\title{
Neue Freiheitsgrade
}

\section{Liebe Leserin, lieber Leser,}

das Wiener Motorensymposium gilt gemeinhin als das Mekka der Motorenentwickler, zumindest in den Eröffnungskeynotes jedoch spielt auch das Thema Gesamtfahrzeug - vor allem vor dem Hintergrund der zunehmenden Elektrifizierung - eine immer wichtigere Rolle.

Effizienz und Kostenersparnis sind auch hier die wesentlichen Treiber. So zeigte Peter Gutzmer von Schaeffler am Beispiel eines aufgeladenen 1,4-1-Grundmotors in Verbindung mit einem 48-V-Hybrid und einem Viergang-Automatikgetriebe eine Effizienzsteigerung von rund $18 \%$ auf. Im Getriebe als Schlüsselkomponente, um Motor und E-Antrieb zu verbinden, wird sich die Ganganzahl, so die Prognose, von heute 7 bis 10 auf 4 bis 6 Gänge reduzieren. Die Elektrifizierung des Antriebs macht auch hier einen systemischen Ansatz notwendig, der die Besonderheiten und Vorlieben der unterschiedlichen Märkte und Kunden, beispielsweise beim Thema Doppelkupplungsgetriebe und CVT, berücksichtigt.

Für Rolf Bulander von Bosch ist die Elektrifizierung keine Konkurrenz zum Verbrennungsmotor. Bits und Bytes machten die Mobilität hingegen effizienter. So kann die 48-V-Technik zum Beispiel in der Kompaktklasse nicht nur den $\mathrm{CO}_{2}$ Ausstoß nachhaltig senken, sondern auch die Partikel um bis zu $30 \%$ und das $\mathrm{NO}_{\mathrm{x}}$ um bis zu $20 \%$ an der Quelle reduzieren. Und ein elektronischer Horizont bei einem Plug-in-Hybriden steigert die Effizienz durch seine Optimierung des automatisierten Fahrverhaltens um bis zu $8 \%$.

Die Verbindung des Elektromotors mit dem Verbrennungsmotor - so die Quintessenz in Wien - führt mithin im kombinierten Vortrieb zu völlig neuen
Möglichkeiten. Dieser Schluss gilt auch für die Architektur des Gesamtfahrzeugs. Die E/E-Architektur stellt natürlich neue Herausforderungen an die Konzeption von Karosserien und Innenräumen. Sie eröffnet auf der anderen Seite aber auch ganz andere Perspektiven und Gestaltungsmöglichkeiten. Der kombinierte Antrieb verändert das Package, die zunehmende Automatisierung des Fahrens und Zahl der Assistenzsysteme die Innenräume der Fahrzeuge. Beides hat erhebliche Auswirkungen auf die Karosserie und das Fahrzeugdesign. Hier entstehen ganz neue Freiheitsgrade.

Freundliche Grüße,

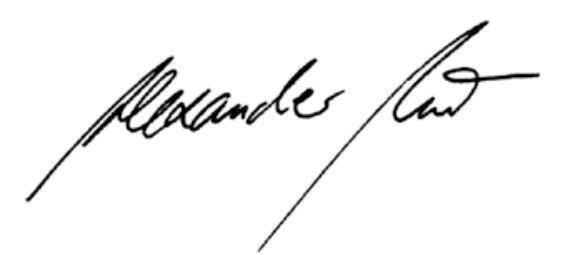

Dr. Alexander Heintzel

Chefredakteur

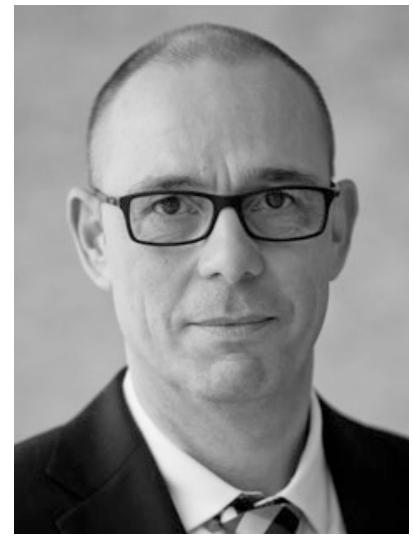

Wir

\section{entwickeln, was bewegt.}

\section{IAV - Ihr Partner \\ für Automotive Engineering}

